\title{
INFLUENCES ON ANALGESIC CHOICES FOR LABOUR AMONG FIRST TIME MOTHERS
}

\author{
Ronald B George MD, Dolores McKeen MD MSc FRCPC, Department of \\ Anesthesia, IWK Health Centre, Halifax, NS, Canada, B3H 3G9
}

INTRODUCTION: Obstetrical analgesia relieves the pain and anxiety of labour. The pain control method chosen is likely influenced by culture, friends, prenatal and intrapartum caregivers. The purpose of this study was to determine what the parturients felt was the single greatest influencing factor that determines the choice of epidural analgesia.

METHODS: With REB approval, all primiparous, singleton gestation, cephalic, labouring women between 34 and 42 weeks who requested epidural analgesia were approached during a one month period. Once epidural analgesia was established consenting patients received the questionnaire.

RESULTS: Of the 140 women recruited, 116 replied (83\% response rate). Only three $(2.6 \%)$ patients saw an anesthesiologist prior to admission. The majority of patients received information regarding labour analgesia management from their friends and family $(83.5 \%)$ and prenatal classes $(75.7 \%)$. Thirty percent had no intention of receiving or preferred to avoid an epidural prior to labour.

During labour, patients were informed of pain management options by the labour nurse in $92.2 \%$ of the cases. An epidural was the most commonly offered analgesic technique $(84.3 \%)$ followed by comfort \& relaxation measures $(77.4 \%)$ and hydrotherapy $(60.9 \%)$. Prior to their epidural $27 \%$ of patients received parenteral narcotics. The majority of women were aware of the major and minor complications associated with epidurals. One hundred percent of the participants felt they were responsible for the choice of labour analgesia and $96.5 \%$ were satisfied with their choice. On a scale of 1-5, friends and family were the greatest influences (median=4, mode $=5$ ) on the choice of an epidural, while the anesthesiologist, obstetrician, and family physician were each the least influential (median=1, mode=1). However, $55.8 \%$ of patients felt a meeting with an anesthesiologist prior to labour would be a benefit.

DISCUSSION: Friends and family are the most influential to parturients selecting a labour epidural. Labour is more painful than expected. At our institution epidural analgesia is the most commonly offered method of pain management. Many women who preferred to avoid an epidural prior to labour had a change of opinion. The majority of primiparous women wish to see an anesthesiologist prior to labour.

\begin{tabular}{l|c|l|c}
\hline \multicolumn{1}{c}{ Variable } & \multicolumn{2}{c}{$\mathbf{n}(\%)$} \\
\hline Age & $29 \pm 10$ years & Ethnicity & \\
\hline Weight & $83.6 \pm 31.6 \mathrm{~kg}$ & $\bullet$ Canadian & $108(93.1)$ \\
\hline Height & $166 \pm 14 \mathrm{~cm}$ & $\bullet$ African Canadian & $3(2.6)$ \\
\hline EGA & $39.7 \pm 2.8 \mathrm{wks}$ & $\bullet$ Other & $5(4.3)$ \\
\hline Cervical Dilation & $4.3 \pm 3.6 \mathrm{~cm}$ & Oxytocin Usage & $91(82.7)$ \\
\cline { 3 - 4 } @ Epidural Insertion & & C/S Rate & $26(23.6)$ \\
\hline
\end{tabular}

